\title{
Neural Cell Type-Specific Expression of QKI Proteins Is Altered in quakingviable Mutant Mice
}

\author{
Rebecca J. Hardy, ${ }^{1}$ Carrie L. Loushin, ${ }^{2}$ Victor L. Friedrich Jr., ${ }^{1}$ Qi Chen, ${ }^{2}$ Thomas A. Ebersole, ${ }^{2}$ \\ Robert A. Lazzarini, ${ }^{1}$ and Karen Artzt ${ }^{2}$ \\ ${ }^{1}$ Brookdale Center for Molecular Biology, Mount Sinai Medical Center, New York, New York 10029, and 2Department of \\ Zoology, University of Texas at Austin, Austin, Texas 78712-1064
}

\begin{abstract}
$q k l$, a newly cloned gene lying immediately proximal to the deletion in the quakingviable mutation, is transcribed into three messages of 5,6 , and $7 \mathrm{~kb}$. Antibodies raised to the unique carboxy peptides of the resulting QKI proteins reveal that, in the nervous system, all three QKI proteins are expressed strongly in myelin-forming cells and also in astrocytes. Interestingly, individual isoforms show distinct intracellular distributions: QKI-6 and QKI-7 are localized to perikaryal cytoplasm, whereas QKI-5 invariably is restricted to the nucleus, consistent with the predicted role of $\mathrm{QKI}$ as an RNA-binding protein. In quakingviable
\end{abstract}

mutants, which display severe dysmyelination, QKI-6 and QKI-7 are absent exclusively from myelin-forming cells. By contrast, QKI-5 is absent only in oligodendrocytes of severely affected tracts. These observations implicate QKI proteins as regulators of myelination and reveal key insights into the mechanisms of dysmyelination in the quakingviable mutant.

Key words: myelination; dysmyelination; nucleus; RNA metabolism; oligodendrocyte; Schwann cell; quaking; STAR proteins quaking viable $\left(q k^{\nu}\right)$ is an autosomal recessive mutation in mice characterized by severe dysmyelination of the CNS (Sidman et al., 1964). CNS myelin of $q k^{v}$ mice exhibits a reduced number of myelin lamellae, a lack of compaction of myelin sheaths, and abnormal production of cytoplasmic loops (Hogan and Greenfield, 1984). In the peripheral nervous system (PNS), the phenotype is relatively mild, although unmyelinated segments of nerve, irregular paranodes, and abnormal Schmidt Lanterman incisures have been described (Suzuki and Zagoren, 1977). The extensive literature on the $q k^{v}$ phenotype over the past 30 years has described many subtle changes in lipid metabolism, myelin protein levels, and even levels of several neurotransmitters in $q k^{v}$ mice, as compared with wild-type mice (reviewed in Hogan and Greenfield, 1984). However, because many of these changes are observed in other dysmyelinating mouse mutants, they most probably reflect secondary changes of the hypomyelinating environment and are not a direct result of the $q k^{v}$ mutation itself. Thus, the primary defect in $q k^{v}$ that leads to the dysmyelinating phenotype has not yet been identified.

$q k I$ is a newly cloned gene that lies $1.1 \mathrm{~kb}$ centrometric to the deletion in the $q k^{v}$ mutation on mouse chromosome 17 (Ebersole et al., 1992, 1996). Transcription of the $q k I$ gene produces three distinct messages of 5,6 , and $7 \mathrm{~kb}$, which differ in their coding regions only at their $3^{\prime}$ ends and in their extensive $3^{\prime}$ untranslated

Received Aug. 6, 1996; revised Sept. 24, 1996; accepted Oct. 1, 1996.

This work was supported by National Institutes of Health Grant NS33165 to V.L.F. and R.A.L. and National Institute of Child Health and Human Development Grants HD10668 and HD30658 to K.A. We thank Stephane Zaffran and Michel Semeriva for sharing their Drosophila sequence before publication. This is manuscript 227 from the Brookdale Center for Molecular Biology.

Correspondence should be addressed to Dr. Rebecca Hardy, Brookdale Center for Molecular Biology, Mount Sinai Medical Center, Box 1126, 1 Gustave L. Levy Place, New York, NY 10029.

Dr. Chen's present address: Genentech, 460 Point San Bruno Boulevard, South San Francisco, CA 94080.

Dr. Ebersole's present address: Department of Genetics, Cambridge University, Cambridge CB2 3EH, UK.

Copyright (C) 1996 Society for Neuroscience $0270-6474 / 96 / 167941-09 \$ 05.00 / 0$ regions. The predicted amino acid sequences of the $q k I$ mRNAs contain a $\mathrm{KH}$ domain that is common to all three isoforms. $\mathrm{KH}$ domains, originally identified in the human heterologous nuclear ribonucleoprotein K protein (Siomi et al., 1993), are shared by a diverse family of proteins that bind to singled-stranded RNA, apparently without sequence specificity, and $\mathrm{KH}$-containing proteins are thought to be involved in the regulation of cellular RNA metabolism (Musco et al., 1996). QKI shares homology to a subset of KH-containing proteins, which includes Sam68, a downstream target of Src, Fyn, and Grb2 (Fumagalli et al., 1994; Taylor and Shalloway, 1994; Lock et al., 1996), and the Caenorhabditis elegans tumor suppressor gene gld-1 (Jones and Schedl, 1995). Thus features of QKI amino acid sequence suggest a role in the regulation of RNA metabolism and possibly also signal transduction, but as yet, experimental evidence for the function(s) of the QKI proteins, is not available.

In this study, we have used specific antibodies to localize each of the three QKI proteins in nervous system tissue. We show that in wild-type mice all three QKI isoforms are abundant in myelinating cells of the central and peripheral nervous system but also are present in astrocytes. By contrast, in $q k^{v}$ mutants, the levels of QKI-6 and QKI-7 are reduced dramatically in myelinating cells, whereas that of the third isoform, QKI-5, is reduced only in severely affected regions of the brain. These observations reveal important insights into the possible function of QKI proteins in wild-type brain and also into the mechanisms of dysmyelination in the $q k^{v}$ mutant.

\section{MATERIALS AND METHODS}

Mice. Wild-type mice were B6D2F1. Mutant mice used were as follows: $q k^{v} / q k^{v}$ mice from the Austin colony [maintained as previously described (Ebersole et al., 1996)], shiverer (shi/shi) mice on agouti background, and jimpy male hemizygotes on the B6C3HF1 background.

Northern blot analysis. Total RNA from mouse brain was isolated by the use of $\mathrm{LiCl} /$ urea (Geliebter, 1987). RNA gels and Northern blots were prepared and hybridized according to standard methods (Ebersole et al., 1996). The same blot was used for hybridization with probes from the 
3'UTR sequences specific for the 5 and $6 / 7 \mathrm{~kb}$ messages. Fragment 0.9 HincII from cDNA $1-2-3$ is unique for the $5 \mathrm{~kb}$ message, whereas fragments 1.4 Bam HI, EcoRI, and 1.7 EcoRI are derived from cDNA 2-3-3 and detect the 6 and $7 \mathrm{~kb}$ messages. For information on these clones, see Ebersole et al. (1996).

QKI antibodies. Three peptides of the unique carboxy tails of QKI-5 (GAVATKVRRHDMRVHPYQRIV TADRAATGN), QKI-6 (GMAFPTKG), and QKI-7 (EWIEMPVMPDISAH) were conjugated to KLH by glutaraldehyde linkage and carbamide chemistry and used to immunize two rabbits for each peptide (Research Genetics, Huntsville, AL). Antisera generated by the duplicate rabbits gave identical immunolabeling results. Antisera were purified by immunoaffinity chromatography with the relevant peptide via the Affigel system (Bio-Rad, Hercules, CA), according to the manufacturer's instructions.

Antibodies. Rat monoclonal antibodies to glial fibrillary acidic protein (GFAP) were a gift of Dr V. M. Lee (Lee et al., 1984). Mouse monoclonal antibodies to myelin basic protein (MBP), neurofilaments, and histones were purchased from Boehringer Mannheim (Indianapolis, IN), Sternberger Monoclonals (Baltimore, MD), and Chemicon (Temecula, CA), respectively. Mouse monoclonal antibodies to GAP-43 were purchased from Sigma (St. Louis, MO). Fluorochrome- and biotin-conjugated secondary antibodies and fluorochrome-conjugated streptavidin were purchased from Jackson Immunologicals (West Point, IL), Southern Biotech (Birmingham, AL), Amersham (Amersham, UK), and Vector (Burlingame, CA).

Immunoblotting. Brain homogenates were made from wild-type mice by homogenizing tissue in $2 \%$ SDS in $20 \mathrm{~mm}$ Tris, $\mathrm{pH} 7.6$ (or without SDS for use on nondenaturing gels). For denaturing gels, $15 \mu \mathrm{g}$ of brain/well was diluted in loading buffer (final concentration: $0.25 \mathrm{M}$ Tris, $\mathrm{pH} 6.8$, $10 \%$ glycerol, $1 \%$ SDS, $100 \mathrm{~mm}$ DTT, and $0.05 \%$ bromophenol blue) and subjected to SDS discontinuous polyacrylamide gel electrophoresis $(10 \%$ polyacrylamide gel). For nondenaturing gels, brain homogenate was diluted in loading buffer in the absence of SDS, centrifuged to remove insoluble proteins, and run on a $10 \%$ polyacrylamide gel in the absence of SDS. For both types of gel, proteins then were transferred to Immobilon P membrane (Millipore, Bedford, MA) and detected by QKI antibodies at 1:10,000-1:50,000 dilution, followed by alkaline phosphataseconjugated donkey anti-rabbit secondary antibody (Jackson Immunologicals) diluted 1:5000, with the nitro blue tetrazolium and 5-bromo-4chloro-3-indolyl phosphate detection system. In peptide inhibition experiments, QKI antisera diluted 1:10,000 was incubated with $1 \mu \mathrm{g}$ of immunizing or nonspecific peptide for $1 \mathrm{hr}$ before incubation with membrane. Immunoreactivity of affinity-purified antisera could be eliminated by previous incubation with $100 \mathrm{ng}$ of specific peptide (data not shown).

Tissue preparation and immunocytochemistry. P14 wild-type, $q k^{v}$, shiverer, or jimpy mutant mice were perfused through the left ventricle with $4 \%$ paraformaldehyde in $0.1 \mathrm{M}$ phosphate buffer, $\mathrm{pH} 7.4$. Tissue to be cryostat-sectioned was dissected out and cryoprotected in $30 \%$ sucrose in $0.1 \mathrm{M}$ phosphate buffer, $\mathrm{pH} 7.4$, overnight at $4^{\circ} \mathrm{C}$, embedded in OCT compound (Miles Scientific, Elkhart, IN), and snap-frozen. Sections $(5-10 \mu \mathrm{m})$ were thaw-mounted onto Superfrost slides (Fisher Scientific, Pittsburgh, PA) and refrozen for storage at $-20^{\circ} \mathrm{C}$.

Tissue sections were treated as follows, depending on the antigen: methanol, $5 \mathrm{~min}$ at $4^{\circ} \mathrm{C}$ (MBP, GAP-43); $0.1 \%$ Triton $\mathrm{X}-100$ in $0.5 \mathrm{M}$ Tris, $\mathrm{pH}$ 7.6, $10 \mathrm{~min}$ at room temperature (GFAP, neurofilaments, histones). Neither treatment was necessary for, nor adversely affected, immunolabeling with QKI antibodies. Then all sections were blocked in $10 \%$ normal goat serum, $1 \%$ gelatin, $5 \%$ BSA, and $0.05 \%$ sodium azide in 0.5

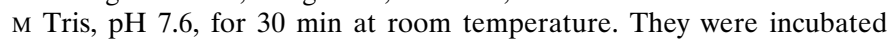
overnight with primary antibodies diluted in the same solution and then in fluorochrome- or biotin-conjugated species or subclass-specific secondary antibodies for $3 \mathrm{hr}$ at room temperature, followed by fluorochromeconjugated streptavidin for $1 \mathrm{hr}$ at room temperature. Each of these steps was followed by three washes in $0.5 \mathrm{M}$ Tris, $\mathrm{pH} 7.6$ (20 min each). QKI primary antibodies were used at a dilution of 1:1000. For peptide inhibition experiments, QKI antisera, at a dilution of 1:1000, were incubated with $1 \mu \mathrm{g}$ of immunizing or nonspecific peptide before overnight incubation with sections. Then sections were mounted on slides and coverslipped with $2.5 \%$ diazabicyclo-octane in glycerol/0.5 M Tris, $\mathrm{pH} 8.6$ (9:1). Slides were analyzed with a Leica TCS confocal microscope. Composite figures were assembled in Adobe PhotoShop and printed on a dye sublimation photoprinter.

\section{RESULTS}

\section{qkl messages are regulated developmentally in brain}

We have demonstrated previously that the three distinct $q k I$ messages are expressed in adult wild-type whole-mouse brain (Ebersole et al., 1996). To determine whether expression levels of these messages are regulated during development, we performed Northern analyses of total RNA from whole brain of wild-type mice aged postnatal day $2(\mathrm{P} 2)$ to adult with probes that detect either the $5 \mathrm{~kb} q k I$ transcript or the 6 and $7 \mathrm{~kb} q k I$ transcripts (Fig. $1 a$ ). We found that, although all three $q k I$ messages were present at each age sampled, the different $q k I$ isoforms had distinct developmental profiles. The $5 \mathrm{~kb}$ transcript is expressed highly in brain throughout the first two postnatal weeks, but levels decline thereafter. By contrast, the 6 and $7 \mathrm{~kb}$ transcripts are expressed throughout postnatal development into adulthood, although expression levels peak at $\sim \mathrm{P} 14$.

\section{QKI antisera}

To determine the localization of each of the three QKI proteins in murine nervous system, we raised rabbit polyclonal antibodies against C-terminal peptides specific to each of the QKI proteins (Fig. 1b). In immunoblot analysis of nondenaturing polyacrylamide gels, antisera to QKI-5 and QKI-6 each strongly recognized distinct bands in homogenates of P14 brain (Fig. 1c). Reactivity against these bands could be eliminated by previous incubation of antisera with immunizing peptide, but not nonspecific peptide (Fig. 1c). Peptide-inhibited antisera gave no immunolabeling on brain sections (see below).

Under denaturing conditions, antisera raised to the C-terminal peptide of QKI-5 reacts with two proteins of 45 and $38 \mathrm{kDa}$ in P14 brain (Fig. 1c). Reactivity against both proteins could be eliminated by preabsorption of QKI-5 antisera with immunizing peptide. However, only the $45 \mathrm{kDa}$ protein was recognized by affinitypurified QKI-5 antisera (data not shown). Because affinitypurified and crude QKI-5 antisera gave identical immunolabeling patterns on frozen brain sections (see below), the significance of the $38 \mathrm{kDa}$ band is not clear at present.

Antisera raised against the C-terminal peptide of QKI-6 reacts with multiple proteins under denaturing conditions (data not shown), presumably because immunoreactive epitopes not available for binding to antibody in native proteins become exposed after denaturation. Because the QKI-6 C-terminal peptide consists of only eight amino acids, cross-reactivity with similar sequences in other proteins is not surprising. However, because the antisera recognize only a tight doublet on native gels, to which reactivity can be competed by immunizing peptide (Fig. 1c), and also exhibit strikingly restricted patterns of immunoreactivity in brain sections (see below), we believe that the antisera is specific for QKI-6 under nondenaturing conditions. Affinity-purified antisera raised against the QKI-7 C-terminal peptide weakly recognized a $43 \mathrm{kDa}$ protein on immunoblots of denaturing gels (data not shown). Both crude and affinity-purified QKI-7 antisera gave similar immunolabeling patterns to QKI-6 antisera, and this immunoreactivity could be eliminated by previous incubation with the QKI-7 immunizing peptide, but not the QKI-6 or QKI-5 peptides (see below).

\section{QKI proteins are abundant in oligodendrocytes}

QKI antisera were used to determine the localization of each of the QKI isoforms in P14 wild-type murine brain. We chose to study P14 animals, because all three $q k I$ transcripts are abundant at this stage of development (Fig. 1a). Parasagittal or coronal 10 


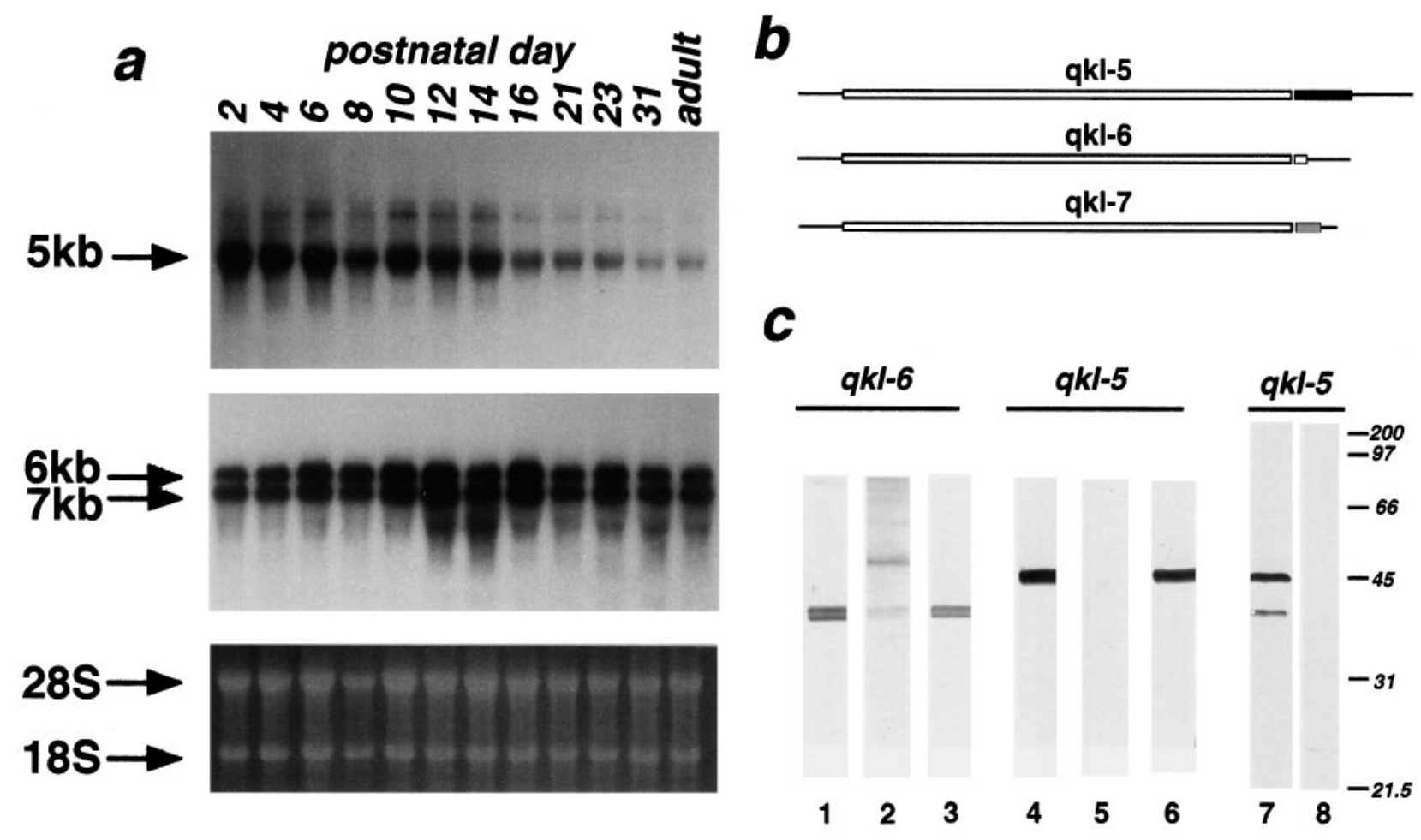

Figure 1. $a$, Northern blot analysis of $q k I$ messages during brain development. A single Northern blot of total brain RNA from whole brain of mice aged $\mathrm{P} 2$ to adult was hybridized with a cDNA probe specific for $q k I 5 \mathrm{~kb}$ transcript and a cDNA that recognizes the $q k I 6$ and $q k I 7 \mathrm{~kb}$ transcripts. Ethidium bromide staining is shown as a loading control. All three $q k I$ messages are expressed throughout postnatal development and into adulthood. Levels of the $5 \mathrm{~kb}$ transcript decline dramatically after the second postnatal week, whereas levels of the 6 and $7 \mathrm{~kb}$ transcripts, although maximal at P14, decline only modestly thereafter. $b$, Production of anti-QKI peptide antisera. Peptides were manufactured with sequences from the C-terminal tails unique to each QKI isoform, as indicated by the shaded areas. c, Immunoblot analysis of QKI antisera with P14 mouse brain. Lanes 1-3, QKI-6, nondenaturing gel; lanes 4-6, QKI-5, nondenaturing gel; lanes 7, 8, QKI-5, denaturing gel. Antisera raised against QKI-6 C-terminal peptide recognize a tight doublet on nondenaturing gels (lane 1). Reactivity for these bands can be eliminated by previous incubation with QKI-6 immunizing peptide (lane 2), but not QKI-5 immunizing peptide (lane 3). Antisera raised against the QKI-5 C-terminal peptide recognize a single, yet distinct, band on nondenaturing gels (lane 4). QKI-5 immunizing peptide (lane 5), but not QKI-6 immunizing peptide (lane 6), can compete away reactivity to this band. The single band recognized by QKI-5 antisera can be resolved into two bands of 45 and $38 \mathrm{kd}$ on SDS-denaturing gels (lane 7). Reactivity to both bands can be competed away by previous incubation with QKI-5 immunizing peptide (lane 8).

$\mu \mathrm{m}$ frozen sections of paraformaldehyde-fixed P14 mouse brain were immunolabeled with each of the QKI antisera. In all cases, antisera raised to QKI-7 peptide gave similar immunolabeling patterns to those raised to QKI-6 peptide, and so for clarity, only results for QKI-6 are mentioned in the text.

QKI immunoreactivity was found throughout the entire brain but was particularly prominent in cells lying in white matter tracts. Double immunolabeling that used antibodies to the MBP and QKI isoforms revealed that $\mathrm{QKI}^{+}$cells are abundant among myelinated axons (Fig. 2). Here, QKI-6 ${ }^{+}$cells have ovoid perikarya and are found in interfascicular rows, characteristic of white matter oligodendrocytes (Fig. $2 b$ ). Figure $2 d$ shows a cell, adjacent to striatal axon bundles, in which QKI-6 is localized to proximal processes contiguous with an $\mathrm{MBP}^{+}$myelin sheath, thus confirming its identity as a myelin-forming oligodendrocyte. In oligodendrocytes, QKI-6 immunoreactivity was concentrated in perikaryal cytoplasm but was also apparent at lower levels in the nucleus. By contrast, QKI-5, which is localized to the same population of cells, was restricted, strikingly, to the nucleus (Fig. 2c). Note that under our labeling conditions the myelin sheaths themselves show no QKI immmunoreactivity.

QKI proteins were detected in oligodendrocytes throughout the brain in both gray and white matter, including cerebellum (Fig. 3) and optic nerve (Fig. 4). All patterns of immunoreactivity seen with crude antisera were identical to those seen with affinitypurified antibodies (data not shown). Preincubation of crude serum with immunizing peptide completely abolished all immunoreactivity on frozen sections, but immunolabeling was unaffected after preincubation of antisera with unrelated peptide.

\section{QKI proteins also are expressed by Bergmann glia and other astrocytes}

In addition to the strong QKI immunoreactivity in oligodendrocytes, we also saw QKI immunolabeling in other CNS cell types. For example, in the cerebellum, the Bergmann glia of the Purkinje cell layer contained levels of QKI-5 and QKI-6 comparable to those in oligodendrocytes (Fig. 3a). Purkinje cells themselves, detected with antibodies to neurofilaments, were devoid of QKI immunoreactivity, as were all other cerebellar neurons. In fact, no immunoreactivity for any of the three QKI proteins was seen in any CNS neurons (Fig. 3c).

To determine whether QKI is localized to other astrocyte populations, we double-labeled sections with antibodies to QKI and the astrocyte protein GFAP. We found that astrocytes of the cerebellum (Figs. 3b, 7a) as well as hippocampus, cerebral cortex, and all white matter tracts (Fig. $3 d-f$ ), including the optic nerve (Fig. 4), all contain moderate amounts of QKI. As in oligodendrocytes, QKI-6 was found in astrocyte cytoplasm as well as in 

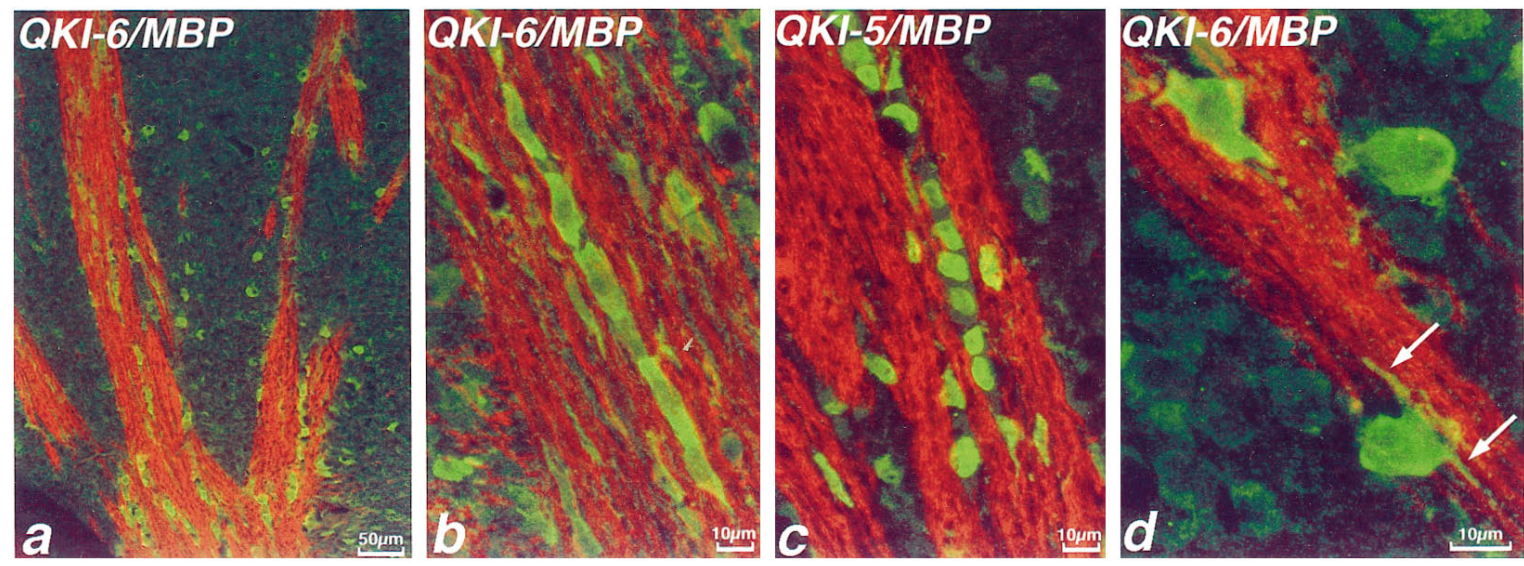

Figure 2. QKI proteins are present in oligodendrocytes in P14 brain. Double immunolabeling of P14 mouse striatum with antisera to MBP (red) and QKI-6 (green, $a, b, d)$ or QKI-5 (green, $c$ ). Numerous QKI- $6^{+}$cells can be seen among the MBP ${ }^{+}$-myelinated fibers of the striatum $(a)$ and at higher magnification $(b)$. These cells are located in rows between myelinated axons, characteristic of interfascicular oligodendrocytes. The same population of cells also is immunolabeled by QKI-5 antisera (c); by contrast, QKI-5 immunoreactivity is restricted to the nucleus. $d$, A cell lying adjacent to myelinated striatal fibers has QKI-6 immunoreactivity in perikaryal cytoplasm but also in proximal processes that are contiguous with an $\mathrm{MBP}^{+}$myelin sheath (arrows).

Figure 3. QKI proteins also are present in astrocytes in P14 brain but are absent from CNS neurons. $a$, Double immunolabeling of P14 mouse cerebellum with antibodies to neurofilaments (NF; red) and QKI-6 (green). QKI-6 immunoreactivity is seen in oligodendrocytes of the cerebellar white matter (arrowheads) but is also found in cells of the Purkinje cell layer. NF immunolabeling demonstrates that, whereas Purkinje neurons $(p)$ are devoid of QKI-6, the adjacent Bergmann glia are QKI-6 ${ }^{+}$. In the cerebellar granule cell layer (gc), granule neurons are QKI-6 ${ }^{-}$, but some weakly QKI- $6^{+}$astrocytes can be seen. $b$, Double immunolabeling with antisera to GFAP (red) and QKI-5 (green) shows that astrocytes of the granule cell layer are also QKI- $5^{+}$. $c$, In the cerebral cortex, oligodendrocytes are strongly QKI-7 ${ }^{+}$(green, arrowheads), whereas astrocytes are more weakly QKI- ${ }^{+}$. Pyramidal neurons visualized by NF immunoreactivity (red) are $\mathrm{QKI}-7^{-} . d-f$, Double immunolabeling of P14 mouse corpus callosum with antisera to QKI-6 (green, $d, f$ ) and GFAP (red, $e, f)$. $\mathrm{GFAP}^{+}$astrocytes contain QKI-6 primarily in the cytoplasm of the perikaryon and proximal processes but also at lower levels in the nucleus.
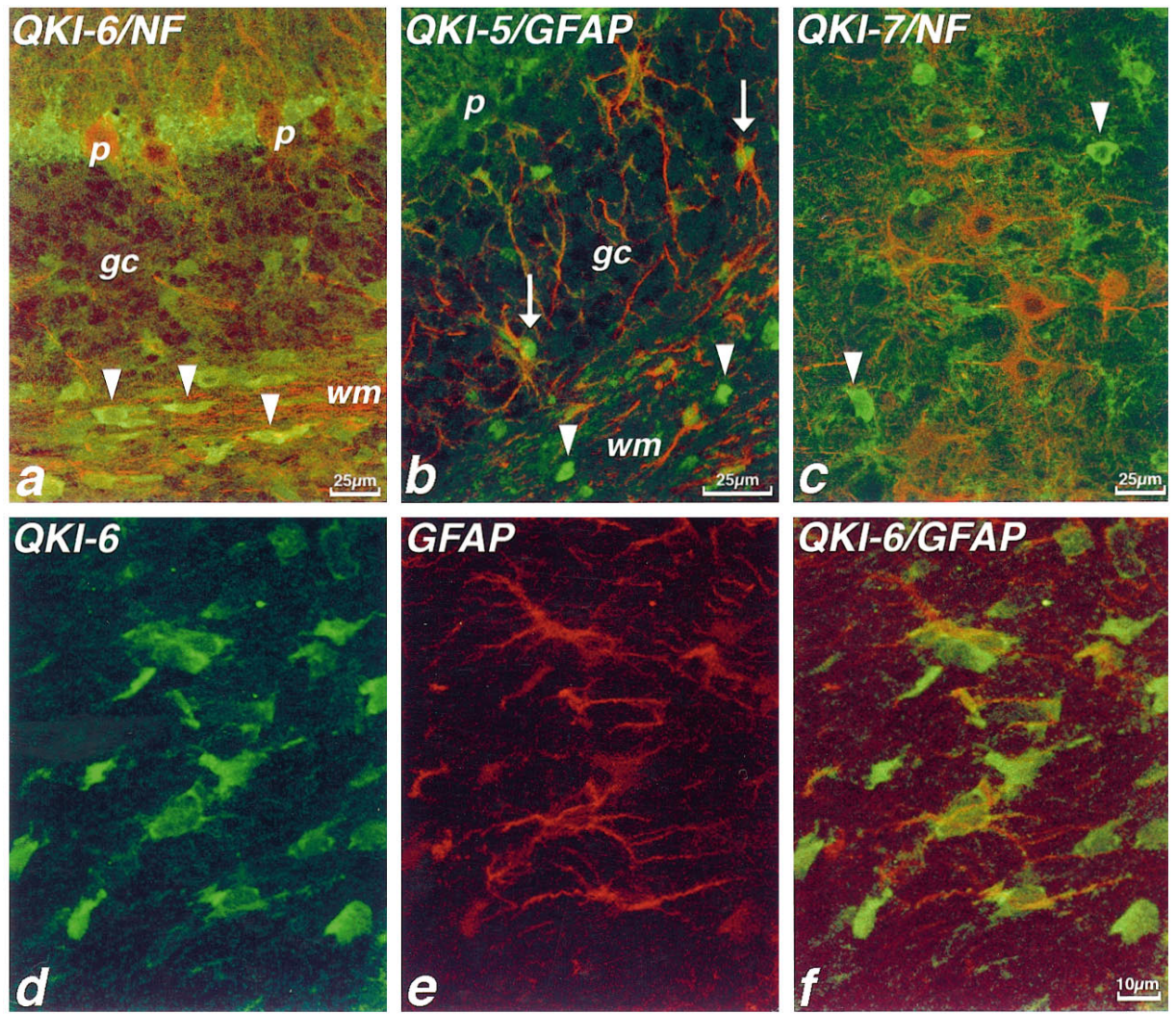

nuclei (Figs. 3d-f, 4a,b). However, QKI-5 immunoreactivity, which in oligodendrocytes is always restricted to the nucleus, was also often found in proximal processes of astrocytes (Fig. 4c,d).

\section{QKI proteins are abundant in myelinating \\ Schwann cells}

Because the QKI proteins are restricted to neuroglia in the CNS, we were interested to see whether they also are expressed in PNS glia. Double immunolabeling with QKI antibodies and antibodies to growth-associated protein 43 (GAP-43), which in peripheral nerve is restricted to nonmyelinating Schwann cells (Curtis et al., 1992), revealed that, whereas QKI-5 and QKI-6 were present at low levels in GAP- $43^{+}$(nonmyelin-forming) Schwann cells, GAP-43- (myelinforming) Schwann cells contained much higher amounts of both QKI-5 and QKI-6 (Fig. 5). As in the CNS, in QKI-containing cells QKI-5 is restricted to nuclei (Fig. 5c), whereas QKI-6 is found primarily in perikaryal cytoplasm (Fig. $5 a$ ).

\section{QKI-6 and QKI-7 proteins are absent from myelin- forming cells in $q k^{v}$ mutants}

$q k I$ transcripts have been detected previously in total brain RNA of $q k^{v}$ mice (Ebersole et al., 1996). This finding is somewhat 


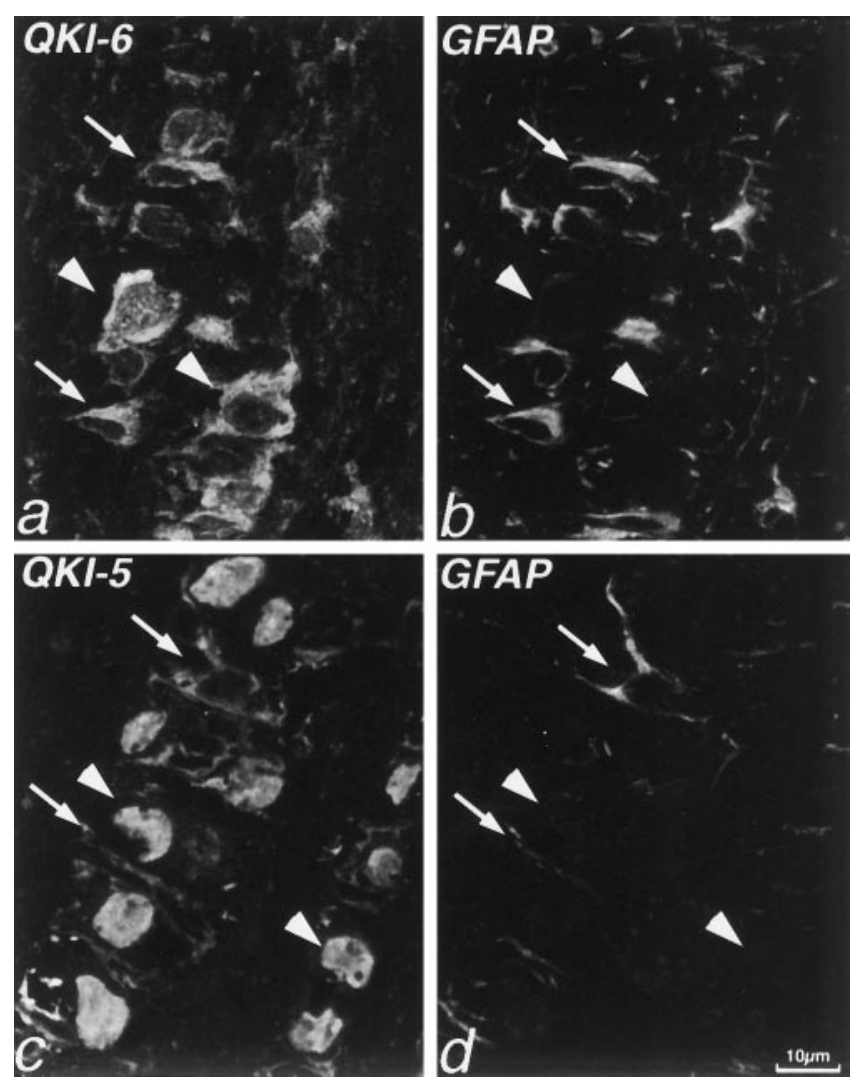

Figure 4. QKI proteins are distributed differentially within oligodendrocytes and astrocytes. Double immunolabeling of P14 mouse optic nerve with antisera against QKI-6 $(a)$ or QKI-5 $(c)$ and GFAP $(b, d)$. $a, b, \mathrm{GFAP}^{-}$oligodendrocytes (arrowheads) contain bright bands of QKI-6 ${ }^{+}$immunoreactivity in their cytoplasm, but some QKI-6 is also present in the nucleus. GFAP ${ }^{+}$astrocytes (arrows) also contain QKI-6, but at lower levels. $c, d$, By contrast, QKI-5 is restricted to the nucleus in $\mathrm{GFAP}^{-}$oligodendrocytes (arrowheads), but in $\mathrm{GFAP}^{+} / \mathrm{QKI}^{-5^{+}}$astrocytes (arrows), QKI-5 immunoreactivity is also present in processes.

surprising, because the $q k^{v}$ deletion lies only $1.1 \mathrm{~kb}$ away from the start of the $q k I$ coding region and therefore might include some of the $q k I$ enhancer/promoter elements. Nevertheless, we thought it possible that distribution of QKI proteins might be affected in $q k^{v}$ mice. On analysis of P14 $q k^{v}$ nervous system, we found dramatic differences in the localization of QKI proteins in homozygous mutants, as compared with their phenotypically normal heterozygous littermates.

In wild-type optic nerve, QKI proteins are found in both oligodendrocytes and astrocytes (Figs. 4, 6a); in fact, almost all cells of normal optic nerve are $\mathrm{QKI}^{+}$(Fig. 6d). By contrast, in $q k^{\nu}$ mice, although astrocytes contain levels of QKI-6 expression comparable to normal mice, $q k^{v}$ oligodendrocytes completely lack detectable levels of QKI-6 (Fig. 6a,b). Previous studies have shown that $q k^{v}$ optic nerve actually contains an elevated number of oligodendrocytes (Friedrich, 1975), and double immunolabeling with antibodies to histones and QKI-6 reveals that there are an increased number of $\mathrm{QKI}^{-}$cells in the optic nerves of $q k^{v}$ mice (Fig. 6, compare $d$ and $e$ ). This suggests that oligodendrocytes are present, but devoid of QKI-6. However, these cells do contain QKI-5. On labeling $q k^{v}$ optic nerve sections, we found that QKI-5 was present in both astrocytes and oligodendrocytes (Fig. $6 c$ ). Indeed, as is the case in normal mice, virtually all of the cells of the $q k^{v}$ optic nerve were QKI-5 ${ }^{+}$(Fig. 6f).
In the cerebellum of $q k^{v}$ mice, QKI-6 is present in astrocytes and also at high levels in Bergmann glia, as is the case in normal littermates (Fig. 7). However, in agreement with data from optic nerve, QKI-6 is not detectable in oligodendrocytes in the cerebellar white matter of $q k^{v}$ mice (Fig. 7, compare $a$ and $b$ ). Our immunocytochemical analysis failed to reveal any detectable levels of QKI-6 and QKI-7 in oligodendrocytes anywhere in the $q k^{v}$ mutant mouse brain.

In the PNS, the dysmyelinating phenotype in $q k^{v}$ mice is mild (Suzuki and Zagoren, 1977). Nevertheless, we observed dramatic changes in localization of QKI proteins similar to those observed in the CNS. In the sciatic nerve of $q k^{v}$ mice, GAP- $43^{-}$(myelinforming) Schwann cells had robust levels of QKI-5 immunoreactivity (Fig. 5g,h). By contrast, both GAP- $43^{+}$and GAP- $43^{-}$ Schwann cells lacked QKI-6 (Fig. 5e,f).

\section{QKI-5 expression varies with severity of dysmyelination}

Hence, in $q k^{v}$ mice, myelin-forming cells of both the CNS and PNS are devoid of QKI-6 and QKI-7, whereas astrocytes seem to have normal levels of both of these QKI isoforms. However, we found that the situation for QKI-5 was more complex and that there was a striking systematic regional variation in the levels of QKI-5 in $q k^{v}$ oligodendrocytes. $q k^{v}$ is unusual among dysmyelinating mouse mutants in that $q k^{v}$ mice exhibit a rostral-caudal gradient in the severity of dysmyelination, with the greatest deficiency of myelin in forebrain tracts, such as the anterior commissure (Friedrich, 1975). We observed that in less severely affected regions, such as the medulla and optic nerve, QKI-5 was present in all oligodendrocytes (Fig. 6). By contrast, in more severely dysmyelinated tracts, such as the corpus callosum and anterior commissure, oligodendrocytes did not exhibit detectable levels of QKI-5, as well as QKI-6 (Fig. 7c,d). It is not yet clear what role, if any, QKI-5 has in this phenomenon; nevertheless, this is the first report of a molecular correlate of the topographic variation in dysmyelination in $q k^{v}$.

\section{Abnormalities in QKI expression are specific to $q \boldsymbol{k}^{\boldsymbol{v}}$ mice}

We wondered whether the changes observed in QKI levels in myelinating cells in $q k^{v}$ mice are a general feature of dysmyelinating mutants. We therefore examined the immunolocalization of QKI proteins in the brains of two other dysmyelinating mouse mutants: shiverer and jimpy. These mutants display a dysmyelinating phenotype as a consequence of mutations in the genes encoding the myelin proteins MBP and proteolipid protein (PLP), respectively (Roach et al., 1983; Dautigny et al., 1986; Nave et al., 1986; Macklin et al., 1987). In both P14 shiverer and jimpy mice, oligodendrocytes contained abundant QKI-5 and QKI-6 immunoreactivity and, as in wild-type mice, QKI-6 was found in the cytoplasm and nucleus, whereas QKI-5 was restricted to the nucleus (Fig. 8). QKI immunoreactivity was found also in shiverer and jimpy Bergmann glia and other types of astrocytes. Thus, our experiments indicate that the shiverer and jimpy mutants exhibit identical QKI localization to wild-type mice. Therefore, the absence of QKI proteins in oligodendrocytes in $q k^{v}$ does not seem to be a general feature of dysmyelinating mutants but is specific to $q k^{v}$ mice.

\section{DISCUSSION}

We have shown that three gene products of the $q k I$ gene are abundant in myelinating cells of the central and peripheral nervous system. These proteins also are located in Bergmann glia and 

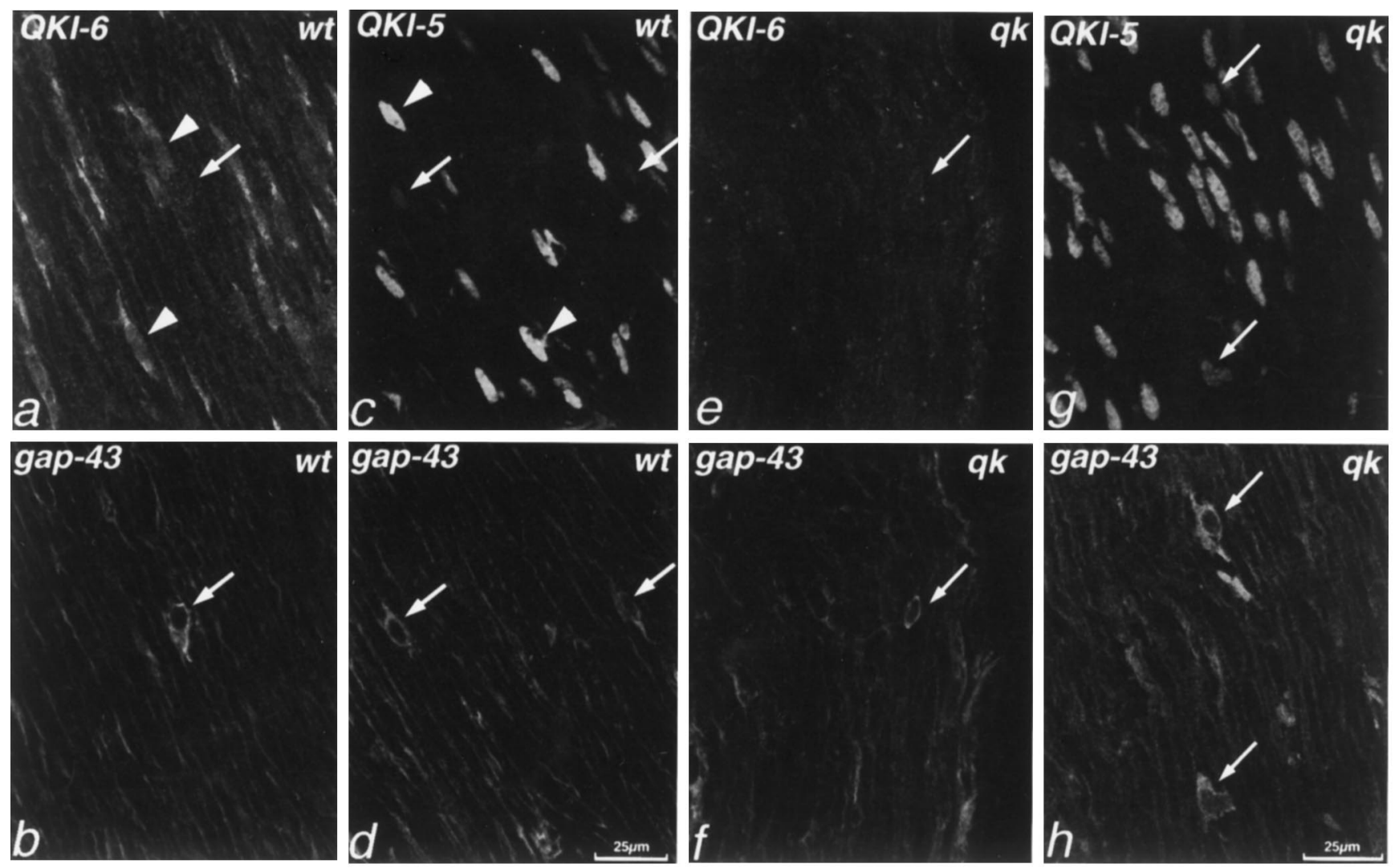

Figure 5. Double immunolabeling of P14 mouse sciatic nerve with antisera against QKI-6 $(a, e)$ or QKI-5 $(c, g)$ and GAP-43 $(b, d, f, h) . a-d$, QKI proteins are present in wild-type peripheral nervous system glia. Both QKI-6 $(a, b)$ and QKI-5 $(c, d)$ are found in GAP-43- myelin-forming Schwann cells (arrowheads) but are absent or barely detectable in GAP- $43^{+}$nonmyelin-forming Schwann cells (arrows). As in the CNS, QKI-5 immunoreactivity is restricted to the nucleus. $e-h$, In $q k^{v}$ mouse sciatic nerve, QKI-6, but not QKI-5, is absent from myelin-forming Schwann cells. $e$, $f$, QKI-6 immunoreactivity is barely detectable in both GAP- $43^{-}$and GAP- $43^{+}$Schwann cells of $q k^{v}$ sciatic nerve. $g, h$, By contrast, in $q k^{v}$, as in wild-type mice, GAP-43myelin-forming Schwann cells contain QKI-5 in their nuclei; lower levels are found in GAP- $43^{+}$nonmyelin-forming Schwann cells. $a-d$, Wild-type, $w t$; $e-h, q k^{v}, q k$.

other astrocytes but are absent from all CNS neurons. In addition, we show that the QKI proteins are distributed differentially within the cell: QKI-6 and QKI-7 are located primarily in the cytoplasm of the perikaryon and proximal processes, with moderate levels within the nucleus, whereas QKI-5 is found almost exclusively in the nucleus. We also have examined the localization of QKI proteins in the dysmyelinating $q k^{v}$ mutant and have found dramatic changes in their levels in myelinating cells: oligodendrocytes and myelin-forming Schwann cells are devoid of QKI-6 and QKI-7. In addition, the presence of QKI-5 in oligodendrocytes correlates with the severity of the dysmyelinating phenotype: in mildly affected regions of the CNS, QKI-5 is abundant in oligodendrocytes, but it is absent in oligodendrocytes of more severely affected tracts. Oligodendrocytes from two other dysmyelinating mouse mutants, shiverer and jimpy, have wild-type levels and distributions of the QKI proteins, suggesting that the effect seen in the $q k^{v}$ mice may be quite specific to that mutation.

\section{QKI proteins and myelination}

Although the physiological function or functions of the QKI proteins are not known at present, the results presented here implicate the QKI proteins as regulators of myelination. All three QKI isoforms are abundant in both oligodendrocytes and myelinating Schwann cells, indicating that they function in both central and peripheral nervous system myelin formation. The regulation of CNS and PNS myelination remains poorly understood, but despite key differences in the molecular composition of CNS and PNS myelin (Norton and Cammer, 1984), oligodendrocytes and Schwann cells have various regulatory mechanisms in common. For example, the POU transcription factor SCIP is known to operate in both oligodendrocytes and myelinating Schwann cells (Collarini et al., 1992; Weinstein et al., 1995). Both oligodendrocytes and myelinating Schwann cells also target MBP proteins to myelin via translocation of MBP mRNA to the myelin sheath (Colman et al., 1982; Trapp et al., 1987; Ainger et al., 1993). By contrast, the MyTI zinc finger transcription factor that binds to the PLP promoter is found primarily in oligodendrocytes (Kim and Hudson, 1992). A potential role for QKI proteins in myelination is likely to be common to both cell types.

However, the widespread expression of $q k I$ mRNAs and proteins indicates that QKI proteins have a still more universal role. $q k I$ mRNAs also can be detected outside the nervous system in heart, lung, and testes (Ebersole et al., 1996), and immunoreactivity for all three QKI proteins can be detected in astrocytes and Bergmann glia, as well as in neuroectodermal cells of the developing neural tube (R.J.H., unpublished observations). Hence, QKI proteins most probably function in multiple tissues both during development and in the mature animal. However, the study of QKI proteins in myelinforming cells, which have many unique features, including a remarkable metabolic burden, may lead to important insights into more universal function(s) of QKI proteins. 

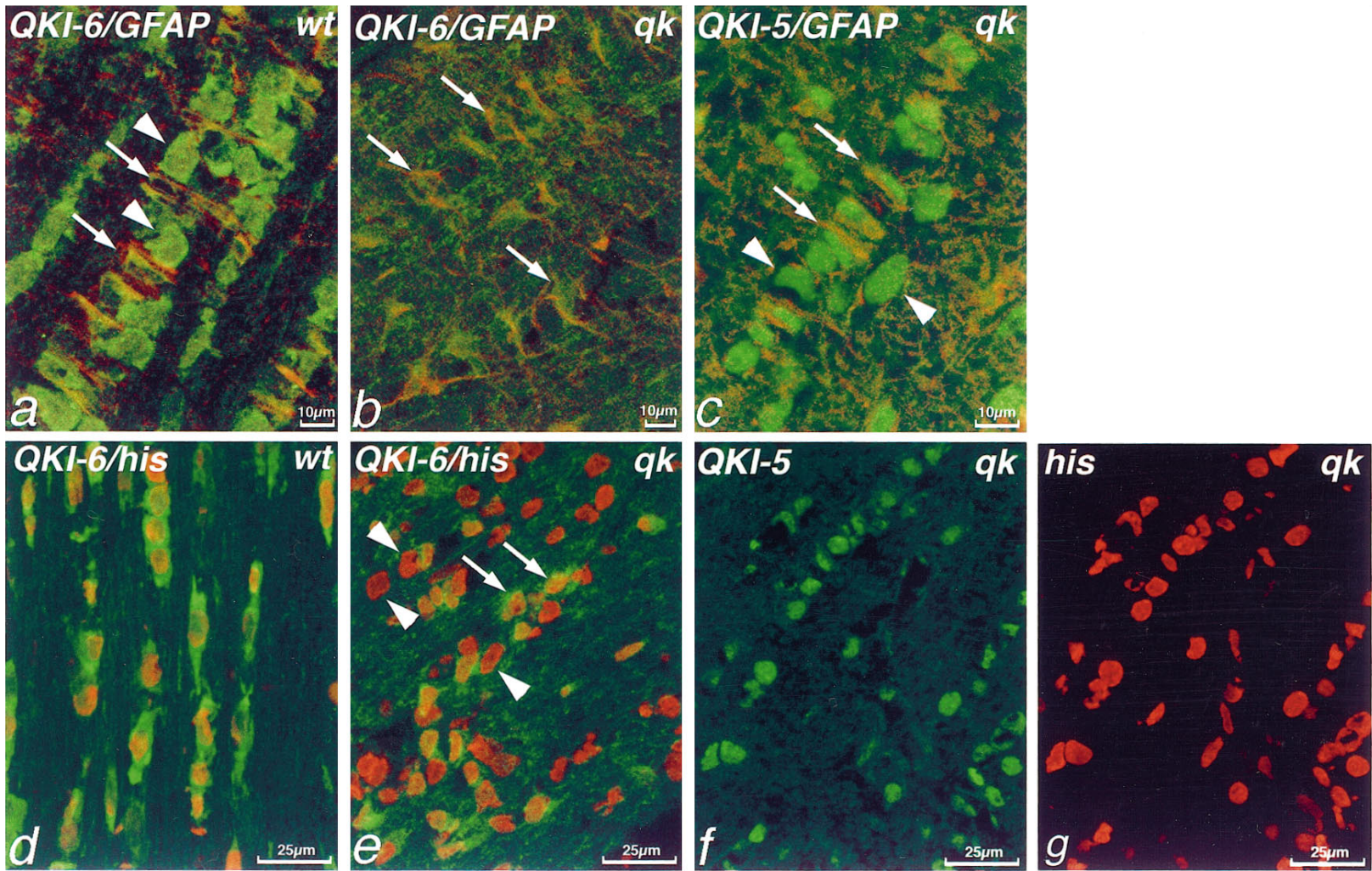

Figure 6. QKI-6, but not QKI-5, is absent from oligodendrocytes in $q k^{v}$ optic nerve. Double immunolabeling of P14 mouse optic nerve with antisera against QKI-6 (green $, a, b, d, e)$ or QKI-5 (green, $c, f)$ and GFAP $(r e d, a-c)$ or histones $(r e d, d, e, g)$. $a$, In wild-type optic nerve, QKI-6 is located in GFAP ${ }^{-}$ oligodendrocytes (arrowheads) as well as GFAP ${ }^{+}$astrocytes (arrows). By striking contrast, in $q k^{v}$ optic nerve $(b)$ QKI-6 immunoreactivity is seen only in $\mathrm{GFAP}^{+}$astrocytes (arrows); oligodendrocytes are devoid of QKI-6. $c$, However, QKI-5 immunoreactivity is present in $q k^{v}$ optic nerve GFAP oligodendrocytes (arrowheads), as well as in $\mathrm{GFAP}^{+}$astrocytes (arrows). In wild-type optic nerve, double labeling with histones reveals that virtually all cells are QKI- $6^{+}(d)$, but in the $q k^{v}$ mutant, many histone ${ }^{+} / \mathrm{QKI}-6^{-}$cells ( $e$, arrowheads) can be seen. As in wild-type, however, virtually all cells in the $q k^{v}$ optic nerve, as labeled with histone antibodies $(g)$, are QKI-5 $(f) . a, d$, wild-type, $w t ; b, c, e, f, g, q k^{v}, q k$.

\section{QKI isoforms are distributed differentially within the cell}

Although in the nervous system all three QKI isoforms are expressed in the same populations of cells, we observed a striking difference in the intracellular distribution of the individual isoforms. In oligodendrocytes and Schwann cells, QKI-5 is always restricted to the nucleus, whereas QKI-6 and QKI-7, although also present in the nucleus, are concentrated in the perikaryal cytoplasm. Because the three isoforms are known to differ only in their C-terminal tails, these probably confer the distinct subcellular localizations observed. For example, the highly basic $\mathrm{C}$ terminus of QKI-5 most probably acts as a nuclear localization signal. The predicted amino acid sequences also indicate that all three QKI isoforms contain a $\mathrm{KH}$ domain, which in other proteins is known to be associated with RNA binding (Musco et al., 1996). The localization of QKI proteins, particularly QKI-5, to the nucleus is consistent with a role for QKI proteins in the regulation of RNA metabolism. Furthermore, QKI-6 and QKI-7 conceivably could be involved in the movement of RNAs from the nucleus to the cytoplasm, because they are present in both compartments.

\section{The $q \boldsymbol{k}^{\mathbf{v}}$ deletion affects QKI protein distribution}

The apparent absence of QKI proteins we have observed in myelinating cells of $q k^{v}$ mice are far more dramatic than any alterations in levels of myelin proteins, mRNAs, or lipid metabolism reported previously for $q k^{v}$ (reviewed in Hogan and Greenfield, 1984). Several observations make it probable that the alter- ations in QKI expression in $q k^{v}$ mice are a direct result of the deletion itself. First, because these changes are not seen in other dysmyelinating phenotypes, they are probably not a secondary event resulting from the hypomyelinated environment. Furthermore, the $q k I$ coding region lies only $1.1 \mathrm{~kb}$ from the deleted sequences in $q k^{v}$, indicating that the regulation of the $q k I$ gene may be affected directly by the deletion. Furthermore, ENUinduced mutations in $q k I$ fail to complement the $q k^{v}$ phenotype (Ebersole et al., 1996).

However, it is not yet clear in molecular terms how the $q k^{\nu}$ deletion leads to alterations of the cell type-specific expression of the QKI proteins or to alterations in the relative levels of the QKI isoforms. One intriguing possibility is that some other protein deleted or truncated by the $q k^{v}$ deletion normally acts as a regulator of $q k I . q k I I$, tentatively identified as a second novel gene and the mRNA of which is truncated in $q k^{v}$ (Ebersole et al., 1996), might be a candidate for such a function. However, because $q k^{v}$ astrocytes have normal levels of QKI, expression in astrocytes presumably is regulated by regions of the chromosome unaffected by the $q k^{\nu}$ deletion. Thus QKI expression may be regulated differentially in distinct cell types. There are precedents for such a mechanism in oligodendrocytes: cell type-specific enhancer elements for the MBP gene have been identified that, alone, can promote MBP expression in oligodendrocytes, but not in Schwann cells (Gow et al., 1992). However, if the $q k^{v}$ mutation included $q k I$ enhancer elements specific to myelin-forming cells, it might be 

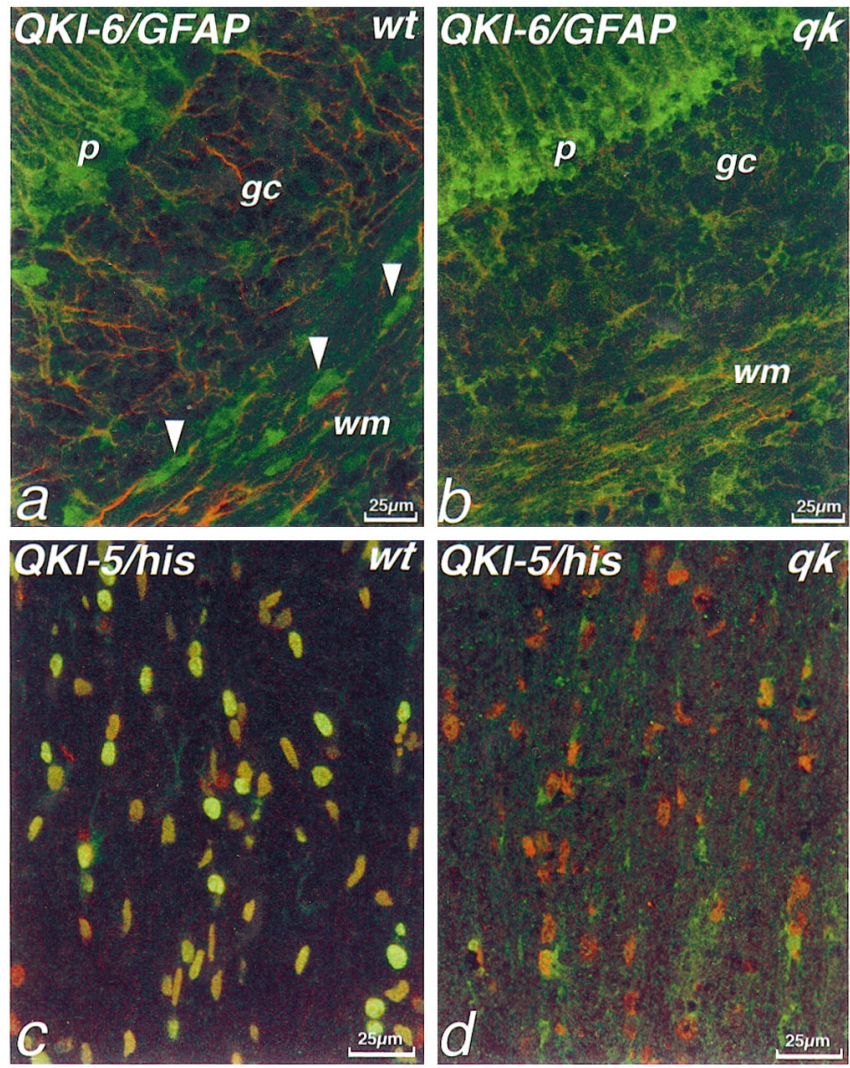

Figure 7. QKI proteins are absent from oligodendrocytes in $q k^{v}$ mice. Double immunolabeling of P14 mouse cerebellum $(a, b)$ or anterior commissure $(c, d)$ with antisera against QKI-6 (green, a, b) and GFAP $($ red, $\mathrm{a}, \mathrm{b})$ or QKI-5 (green, $c, d)$ and histones $(r e d, c, d)$. In wild-type cerebellum, antisera against QKI-6 label the Bergmann glia of the Purkinje cell layer $(p)$, some $\mathrm{GFAP}^{+}$astrocytes in the granule cell layer $(g c)$ and white matter (wm), as well as GFAP ${ }^{-}$oligodendrocytes (arrowheads). By contrast, in $q k^{v}$ cerebellum $(b)$, no QKI-6 immunoreactivity is seen in oligodendrocytes in the white matter, although $\mathrm{GFAP}^{+}$astrocytes and Bergmann glia still are immunolabeled. In wild-type anterior commissure (c), virtually all cells, as determined by anti-histone immunolabeling, also contain QKI-5. However, in anterior commissure from $q k^{v}$ mice $(d)$, only the occasional cell is QKI-5 ${ }^{+}$; the vast majority of histone ${ }^{+}$cells lacks detectable levels of QKI-5. $a, c$, Wild-type, $w t ; b, d, q k^{v}, q k$.

expected that the levels of all three QKI isoforms could be affected. This is not the case; in the PNS and in many CNS regions, levels of QKI-6 and QKI-7 are reduced in myelin-forming cells, whereas levels of QKI-5 are normal. Therefore, $q k^{v}$ mice exhibit alterations in the steady-state levels of individual QKI isoforms exclusively in myelin-forming cells, possibly arising from altered splicing or translation of $q k I$ mRNA or differential stability of QKI polypeptide isoforms.

\section{$\boldsymbol{q} \boldsymbol{k}^{\mathbf{V}}$ mice exhibit abnormalities in RNA metabolism}

The dramatic reduction in QKI expression in $q k^{v}$ myelin-forming cells is intriguing in light of the putative role of QKI as an RNA binding protein and several previous reports of abnormalities in RNA metabolism in $q k^{v}$ mice. First, myelin-associated glycoprotein (MAG), which is found in both CNS and PNS myelin-forming cells, displays several abnormalities in $q k^{v}$ mice, most notably alterations in levels of alternatively spliced isoforms (Frail and Braun, 1985; Braun et al., 1990), and recent evidence suggests that processing and/or turnover of MAG transcripts is affected by the $q k^{v}$ mutation (Bartoszewicz et al., 1995; Bo et al., 1995). Further-
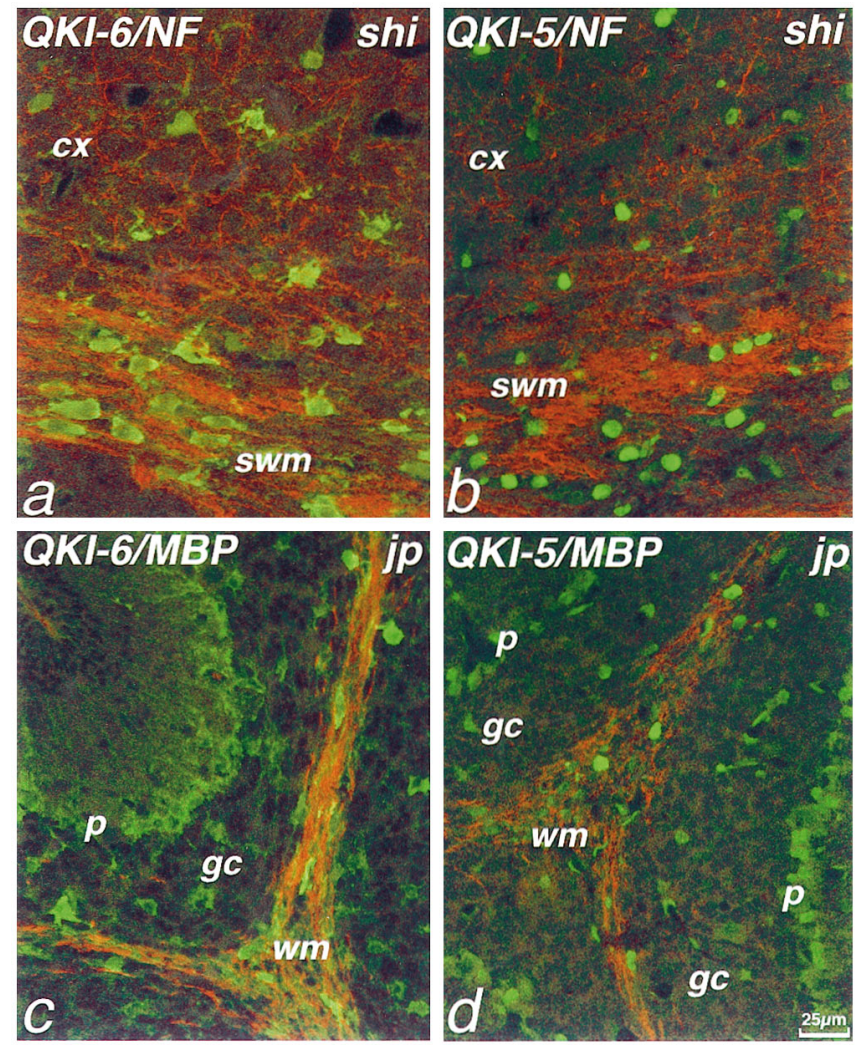

Figure 8. QKI proteins are present in both oligodendrocytes and astrocytes in shiverer and jimpy mutant mice. Double immunolabeling of P14 shiverer $(a, b)$ or jimpy $(c, d)$ mice with antibodies to QKI-6 (green, $a, c)$ or QKI-5 (green, $b, d)$ and NF (red, $a, b)$ or MBP (red, $c, d$ ). $a$, As in wild-type mice, oligodendrocytes of the subcortical white matter $(\mathrm{swm})$ and cerebral cortex $(c x)$ of shiverer mice are immunolabeled strongly with antisera to QKI-6 (a) and QKI-5 (b). Similarly, in jimpy mice, cerebellar white matter oligodendrocytes are immunolabeled strongly by QKI-6 (c) and QKI-5 (d) antisera. Bergmann glia of the Purkinje cell layer $(p)$ and astrocytes of the granule cell layer $(g c)$ also are immunolabeled.

more, proportions of alternatively spliced MBP mRNAs also are affected in $q k^{v}$ mice (Carnow et al., 1984). Second, because of a post-transcriptional event, there is a decrease in PLP mRNA levels in $q k^{v}$ compared with wild-type mice (W. Macklin, personal communication). Third, Barbarese (1991) has reported an intriguing observation that cultured $q k^{v}$ oligodendrocytes are unable to translocate MBP mRNA to their processes, although transport of other mRNAs seemed unaffected. It is possible that some or all of these defects in RNA metabolism may be a consequence of the lack of QKI proteins in $q k^{v}$ myelin-forming cells, resulting in the dysmyelinating phenotype that was observed.

\section{REFERENCES}

Ainger K, Avossa D, Morgan F, Hill SJ, Barry C, Barbarese E, Carson JH (1993) Transport and localization of exogenous myelin basic protein mRNA microinjected into oligodendrocytes. J Cell Biol 123:431-441.

Barbarese E (1991) Spatial distribution of myelin basic protein mRNA and polypeptide in quaking oligodendrocytes in culture. J Neurosci Res 29:271-281.

Bartoszewicz ZP, Noronha AB, Fujita N, Sato S, Bo L, Trapp BD, Quarles RH (1995) Abnormal expression and glycosylation of the large and small isoforms of myelin-associated glycoprotein in dysmyelinating quaking mutants. J Neurosci Res 41:27-38.

Bo L, Quarles RH, Fujita N, Bartoszewicz Z, Sato S, Trapp BD (1995) Endocytic depletion of L-MAG from CNS myelin in quaking mice. J Cell Biol 131:1811-1820. 
Braun PE, Horvath E, Edwards AM (1990) Two isoforms of myelinassociated glycoprotein accumulate in quaking mice: only the large polypeptide is phosphorylated. Dev Neurosci 12:286-292.

Carnow TB, Carson JH, Brostoff SW, Hogan EL (1984) Myelin basic protein gene expression in quaking, jimpy, and myelin synthesis-deficient mice. Dev Biol 106:38-44.

Collarini EJ, Kuhn R, Marshall CJ, Monuki ES, Lemke G, Richardson WD (1992) Downregulation of the POU transcription factor SCIP is an early event in oligodendrocytes differentiation in vitro. Development (Camb) 116:193-200.

Colman DR, Kreibich G, Frey AB, Sabatini DD (1982) Synthesis and incorporation of myelin polypeptides into CNS myelin. J Cell Biol 95:598-608.

Curtis R, Stewart HJ, Hall SM, Wilkin GP, Mirsky R, Jessen KR (1992) GAP-43 is expressed by nonmyelin-forming Schwann cells of the peripheral nervous system. J Cell Biol 116:1455-1464.

Dautigny A, Mattei MG, Morello D, Alliel PM, Pham Dinh D, Amar L, Arnaud D, Simon D, Mattei JF, Guenet JL, Jolles P, Avner P (1986) The structural gene coding for myelin-associated proteolipid protein is mutated in jimpy mice. Nature 321:867-869.

Ebersole T, Rho O, Artzt K (1992) The proximal end of mouse chromosome 17: new molecular markers identify a deletion associated with quakingviable. Genetics 131:183-190.

Ebersole TA, Chen Q, Justice MJ, Artzt K (1996) The quaking gene product necessary in embryogenesis and myelination combines features of RNA binding and signal transduction proteins. Nat Genet 12:260-265.

Frail DE, Braun PE (1985) Abnormal expression of the myelinassociated glycoprotein in the central nervous system of dysmyelinating mutant mice. J Neurochem 45:1071-1075.

Friedrich VL (1975) Hyperplasia of oligodendrocytes in quaking mice. Anat Embryol (Berl) 147:259-271.

Fumagalli S, Totty NF, Hsuan JJ, Courtneidge SA (1994) A target for Src in mitosis. Nature 368:871-874.

Geliebter J (1987) Dideoxynucleotide sequencing of RNA and uncloned cDNA. Focus 9:5-6.

Gow A, Friedrich Jr VL, Lazzarini RA (1992) Myelin basic protein gene contains separate enhancers for oligodendrocyte and Schwann cell expression. J Cell Biol 119:605-616.

Hogan EL, Greenfield S (1984) Animal models of genetic disorders of myelin. In: Myelin (Morrell P, ed), pp 489-534. New York: Plenum.

Jones AR, Schedl T (1995) Mutations in gld-1, a female germ cell-specific tumor suppressor gene in Caenorhabditis elegans, affects a conserved domain also found in Src-associated protein Sam68. Genes Dev 9:1491-1504.

Kim JG, Hudson LD (1992) Novel member of the zinc finger superfamily: a C2-HC finger that recognizes a glia-specific gene. Mol Cell Biol 12:5632-5639.

Lee VM, Page CD, Wu HL, Schlaepfer WW (1984) Monoclonal antibodies to gel-excised glial filament protein and their reactivity with other intermediate filament proteins. J Neurochem 42:25-32.

Lock P, Fumagalli S, Polakis P, McCormick F, Courtneidge S (1996) The human p62 cDNA encodes Sam68 and not the RasGAP-associated p62 protein. Cell 84:23-24.

Macklin WB, Gardinier MV, King KD, Kampf K (1987) An AG-GG transition at a splice site in the myelin proteolipid protein gene in jimpy mice results in the removal of an exon. FEBS Lett 223:417-421.

Musco G, Stier G, Joseph C, Antonietta M, Morelli C, Nilges M, Gibson T, Pastore A (1996) Three-dimensional structure and stability of the $\mathrm{KH}$ domain: molecular insights into the fragile $\mathrm{X}$ syndrome. Cell $85: 237-245$.

Nave KA, Lai C, Bloom FE, Milner RJ (1986) jimpy mutant mouse: a 74-base deletion in the mRNA for myelin proteolipid protein and evidence for a primary defect in RNA splicing. Proc Natl Acad Sci USA 83:9264-9268.

Norton W, Cammer W (1984) Isolation and characterization of myelin. In: Myelin (Morrell P, ed), pp 147-195. New York: Plenum.

Roach A, Boylan K, Horvarth S, Prusiner SB, Hood L (1983) Characterization of cloned cDNA representing rat myelin basic protein: absence of expression in brain of shiverer mutant mice. Cell 34:799-806.

Sidman RL, Dickie MM, Appel SH (1964) Mutant mice (quaking and jimpy) with deficient myelination in the central nervous system. Science 144:309-312.

Siomi H, Matunis MJ, Michael WM, Dreyfuss G (1993) The pre-mRNA binding $\mathrm{K}$ protein contains a novel evolutionarily conserved motif. Nucleic Acids Res 21:1193-1198.

Suzuki K, Zagoren JC (1977) quaking mouse: an ultrastructural study of the peripheral nerves. J Neurocytol 6:71-84.

Taylor SJ, Shalloway D (1994) An RNA-binding protein associated with Src through its SH2 and SH3 domains in mitosis. Nature 368:867-871.

Trapp BD, Moeuch T, Pulley M, Barbosa E, Tennekoon G, Griffith J (1987) Spatial segregation of mRNA encoding myelin-specific proteins. Proc Natl Acad Sci USA 84:7773-7777.

Weinstein D, Burrola PG, Lemke G (1995) Premature Schwann cell differentiation and hypermyelination in mice expressing a targeted antagonist of the POU transcription factor SCIP. Mol Cell Neurosci 6:212-229. 